\section{Measurement of elasticity of normal placenta using the Virtual Touch quantification technique}

\author{
Size $\mathrm{Wu}^{1}$, Ruixia Nan ${ }^{1}$, Yueping $\mathrm{Li}^{2}$, Xiaojing Cui ${ }^{1}$, Xian Liang ${ }^{1}$, Yanan Zhao ${ }^{1}$ \\ Departments of ${ }^{1}$ Ultrasound, and ${ }^{2}$ Obstetrics and Gynecology, Affiliated Hospital of Hainan \\ Medical College, Haikou, China
}

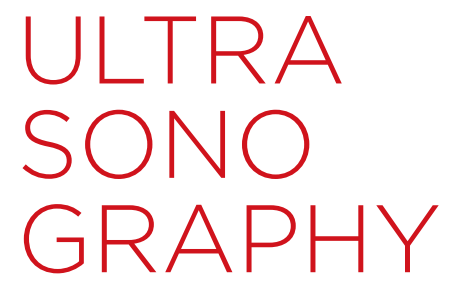

ORIGINAL ARTICLE

http://dx.doi.org/10.14366/usg. 16002 pISSN: 2288-5919 - elSSN: 2288-5943 Ultrasonography 2016;35:253-257

Received: January 13, 2016

Revised: March 31, 2016

Accepted: April 2, 2016

Correspondence to:

Ruixia Nan, MD, Department of Ultrasound, Affiliated Hospital of Hainan Medical College, No. 31, Longhua Road, Haikou 570102, China

Tel. +86-0898-66774347

Fax. +86-0898-66508833

E-mail: nan071051@163.com

This is an Open Access article distributed under the terms of the Creative Commons Attribution NonCommercial License (http://creativecommons.org/ licenses/by-nc/3.0/) which permits unrestricted noncommercial use, distribution, and reproduction in any medium, provided the original work is properly cited.

Copyright @ 2016 Korean Society of Ultrasound in Medicine (KSUM)

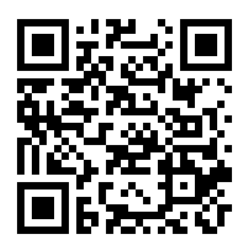

The placenta is a unique organ that forms the biologic interface between the mother and fetus. The importance of the placenta in the lifelong health of both the pregnant woman and the fetus has been underscored [1-4]. However, knowledge about placental structure and function is limited, and the placenta is the least understood human organ [1]. Existing techniques essentially rely on post-delivery examination of the placenta, and typically focus only on molecular and histological investigation [1]. A new approach for assessment of the placenta in vivo would be a significant finding.

\section{Introduction}


Although ultrasonography (US) combined with the Doppler technique can investigate some malformations in the development, positioning, and vasculature of the placenta, which have profound implications for both maternal and fetal well-being, there are still many unknown aspects of the placenta that remain to be uncovered by US technique. Ultrasound elasticity imaging is a novel technique that can determine tissue elasticity or stiffness. Elastography has been used for the differentiation of benign and malignant tumors, the identification of liver fibrosis, and other activities [5-12]. The acoustic radiation force impulse (ARFI) technique is one of the techniques for ultrasound elasticity imaging, which uses the acoustic radiation force through one focalized ultrasound beam to slightly displace the target tissue at the focal spot according to Hooke's law $[5,6]$. The transducer then switches automatically into imaging mode and detects displacements of the focal spot by tracking of the US signal (called speckle). US speckle tracking allows for correlation of the US signal frame by frame in order to detect the displacement of a target tissue with sensitivity of less than a micrometer [5-7]. Methods have been developed that provide "point" measurements, reporting average shear wave speed (and/or Young's modulus) in a local region of interest (ROI). These methods estimate the speed of shear wave propagation through tissues, which is related to the underlying material stiffness through an equation $[5,6]$.

This novel technique has been used for the evaluation of the elasticity of several organs and tissues, but there has been little attention directed toward its role in the assessment of the placenta [8-14]. The aim of this study was to investigate normal placenta elasticity using the Virtual Touch quantification (VTQ) technique, and to identify potential for improving the evaluation of the placenta.

\section{Materials and Methods}

\section{Study Population}

A prospective study on the determination of the elasticity of a normal placenta was conducted between March 2015 and November 2015, in which 1,269 consecutive pregnant women (not including follow-up visits) undergoing routine evaluation of the mother and fetus were considered. Exclusion criteria included previous history of abnormal fetus, placenta, and/or uterus, a known medical disease such diabetes, hypertension, nephritis, or congenital heart disease, and multiple gestation, abnormal fetal growth, or abnormal amniotic fluid index. Participants with an Apgar score of lower than 7 in 5 minutes after birth were excluded in the subsequent analysis. The Apgar score is an index used to evaluate the condition of a newborn infant based on a rating of 0,1 , or 2 for each of the five characteristics of body color, heart rate, response to stimulation of the sole of the foot, muscle tone, and respiration.
In the term infant and late-preterm infant, a 5-minute Apgar score of 7-10 is defined as reassuring, a score of $4-6$ as moderately abnormal, and a score of $0-3$ as low. Because VTQ cannot measure regions deeper than $8.0 \mathrm{~cm}$, and a placenta located at the posterior wall of the uterus in the third trimester was usually deeper than 8.0 $\mathrm{cm}$, pregnant women with a placenta at the posterior wall of the uterus were not included. Pregnant women who showed normal condition after physical examination, laboratory tests, ultrasound, and Doppler evaluation were included for placenta VTQ.

\section{Virtual Touch Tissue Quantification and Data Processing}

A Siemens Acuson S2000 ultrasound system (Siemens Healthcare, Ultrasound Business Unit, Mountain View, CA, USA) was used to acquire shear wave data via the Virtual Touch tissue quantification software package. It was performed by a sonologist with 2 years of experience in the ARFI technique. If grayscale US and Doppler US of the uterus, fetus, and placenta yielded no abnormal findings, the ARFI technique with VTQ was performed in the placenta. A rectangular ROI, void of identifiable blood pools or abnormal echoic regions, was set with a fixed dimension of $1.0 \mathrm{~cm} \times 0.5 \mathrm{~cm}$ with the convex probe (4C1). Shear wave velocity (SWV) was measured in several different ROIs for each placenta. This device generated shear waves by means of short push pulses (4 C1; mean push pulse, 2.67 $\mathrm{MHz}$ ). Pregnant women were supine and breathing normally when measurements were performed.

SWV measurements in placentas located anterior and lateral to the uterus were performed in the supine position. Seven valid measurements of SWV were performed in each placenta with transducer $4 \mathrm{C} 1$ deeper than $1.5 \mathrm{~cm}$ from the probe (depth range, 1.5 to $8.0 \mathrm{~cm}$ ). The measurements were performed at different locations to sample different areas of the placenta. Measurements were performed 3-4 times in each location, the interval between measurements was 2-4 seconds, and the mean SWVs were calculated without the highest and lowest values of measurements in each region. When the velocity measurement was unreliable, the machine automatically displayed figure of velocity measurement, which was thus not taken into consideration in SWV calculations. The number of non-valid measurements was not recorded. Measurements were repeated until the necessary number of reliable values for a complete examination was obtained. SWV means and standard deviations were calculated for each placenta in each patient; the results were expressed in meters per second $(\mathrm{m} / \mathrm{sec})$. To determine intra-observer reliability of the SWV measurement, the ultrasonologist measured the SWV at five sites in 10 third-trimester pregnant women before regular measurement. 


\section{Ethical Statements}

All procedures followed were in accordance with the ethical standards of the responsible committee on human experimentation (institutional and national) and with the World Medical Association Declaration of Helsinki (revised in 2000). The study was approved by the Institutional Ethics Committee, with the recommendation that the SWV measurement should be performed quickly so as to reduce exposure time of the fetus to ARFI, and informed consent was obtained from all patients before inclusion in the study.

\section{Statistical Analysis}

Maternal age and SWV data were expressed as mean \pm SD, and statistical comparison of maternal age between the second and third trimester was performed using a paired samples t test (twotailed). Statistical comparison of SWV between the second and third trimester was performed with the independent samples t test. The intra-observer agreement for operator repeatability was performed using a scale reliability analysis. $A$ t value of $P<0.05$ was considered significant. Statistical analyses were performed with SPSS ver. 20 statistical software (IBM Co., Armonk, NY, USA).

\section{Results}

One hundred and thirty-four pregnant women underwent VTQ evaluation of the placenta; they were followed to delivery. Five pregnant women with neonates who had an Apgar score lower than 7 in 5 minutes at labor, three pregnant women who had delayed delivery of placenta, and five pregnant women who delivered at other institutions were dropped from the study. The other 121 pregnant women with no abnormality in maternity, placenta, fetus, or neonate were included. For convenience of analysis, not all of the 121 pregnant women were included in the final study. The final study population consisted of 50 women in the second trimester (27.94 \pm 4.31 years) and 50 women in the third trimester $(29.2 \pm 4.4$ years) selected randomly from the above 121 pregnant women, and the gestation age at examination was from 14 to 40 weeks (second trimester, $21.1 \pm 3.8$ weeks; third trimester, $33.3 \pm 2.9$ weeks). The SWV of the placenta was $0.983 \pm 0.260 \mathrm{~m} / \mathrm{sec}$, and the minimal and maximal speed was $0.63 \mathrm{~m} / \mathrm{sec}$ and $1.84 \mathrm{~m} / \mathrm{sec}$, respectively. There was no significant difference between second and third trimester of VTQ of the placenta in terms of SWV $(0.978 \pm 0.255 \mathrm{~m} /$ sec vs. $0.987 \pm 0.266 \mathrm{~m} / \mathrm{sec}, P=0.711)$. The maternal age between second and third trimester was $27.9 \pm 4.3$ years and $29.2 \pm 4.4$ years, respectively; there was no significant difference between them $(P=0.159)$. The intra-observer agreement value was 0.638 ( $95 \%$ confidence interval, 0.432 to 0.974 ); there was no significant difference among different measurements $(P=0.072)$. The lower and higher speeds of SWV were measured as almost evenly distributed in placentas located both at the anterior wall and lateral wall of the uterus. A comparison of maternal age and VTQ of the placenta between the second and third trimester is listed in Table 1. Fig. 1 illustrates the placental elasticity measurement using VTQ of the ARFI technique. Fig. 2 illustrates that the distribution of measurements of SWV is almost even at different gestational ages at examination. Fig. 3 illustrates that the distribution of measurements of SWV is almost even at different depths of sample.

\section{Discussion}

Shear wave elastography is based on the concept that shear waves move faster through stiffer regions in a tissue; thus, measurement of SWV allows quantification of placenta stiffness and, indirectly, placental histopathological abnormalities $[5,6]$. The intra-observer agreement value in this study of $0.638(P=0.072)$ indicates a high reliability of the SWV measurement of the placenta using the ARFI technique. However, the P-value is 0.072 , which indicates that it has marginal significance. Theoretically, the placenta in late gestation

Table 1. Comparison of maternal age and VTQ of the placenta between second and third trimester

\begin{tabular}{lccc}
\hline \multicolumn{1}{c}{ Category } & Maternal age $(\mathrm{yr})$ & VTQ & $\begin{array}{c}\text { Minimum- } \\
\text { maximum of VTQ }\end{array}$ \\
\hline $\begin{array}{l}\text { Second trimester } \\
(\mathrm{n}=50)\end{array}$ & $27.94 \pm 4.31$ & $0.978 \pm 0.255$ & $0.63-1.81$ \\
$\begin{array}{l}\text { Third trimester } \\
(\mathrm{n}=50)\end{array}$ & $29.18 \pm 4.42$ & $0.987 \pm 0.266$ & $0.66-1.84$ \\
P-value & 0.159 & 0.711 & - \\
\hline
\end{tabular}

VTQ, Virtual Touch quantification.

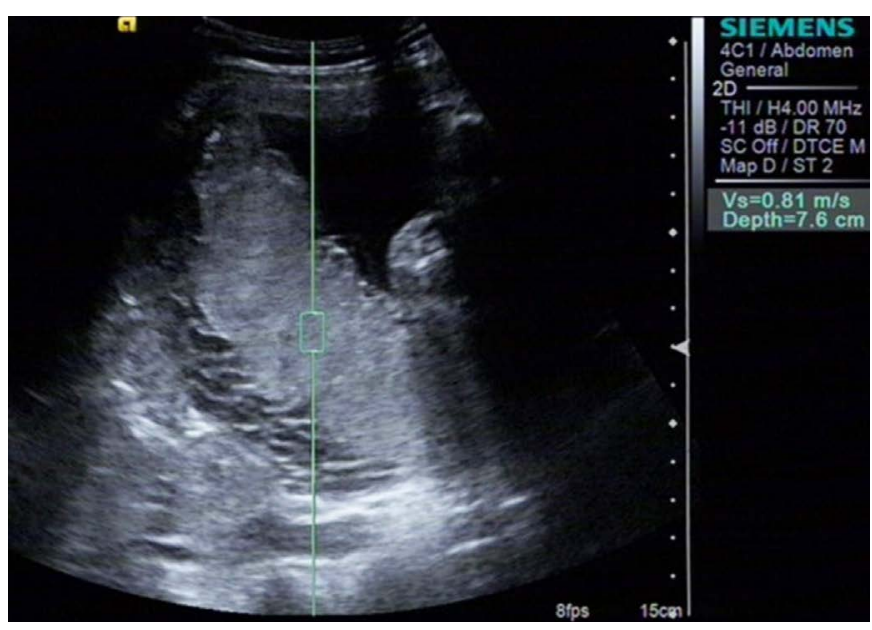

Fig. 1. A 27-year-old woman in the third trimester at 31 weeks. The placental elasticity was determined using Virtual Touch quantification of the acoustic radiation force impulse technique, setting the sampling region in different regions of the placenta. 


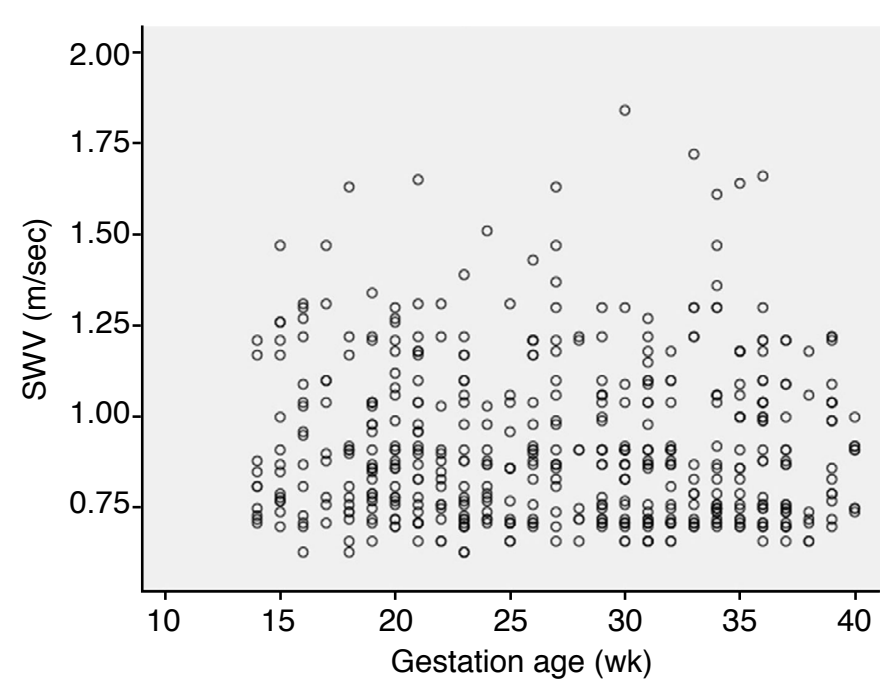

Fig. 2. Scatter plot of measurements of shear wave velocity (SWV) and gestational age at examination. The distribution of measurements of SWV is almost even at different gestational ages at examination.

may be stiffer than at an earlier time in the evolution of the mature placenta, but in this study, the VTQ showed no significant difference between second trimester and third trimester. Meanwhile, we found that the placenta varied largely in the measurement of VTQ, even in the same gestational age at examination (Fig. 2). This is similar to the fact that the calcification of the placenta occurs independently from gestational age at examination after second trimester $[15,16]$. Our results are similar to the results showing that measurements of placental VTQ varied largely in vivo by Li et al. [11]. Ohmaru et al. [14] and ex vivo by Sugitani et al. [13]. The VTQ values determined in vivo are obviously lower than Sugitani et al. [13] found ex vivo. Large VTQ variance also exists in the measurements of other organs [8-10], and large placental VTQ variance may be a common phenomenon. Previous studies $[5,6]$ show that depth affects the measurement of tissue VTQ, but in this study, in spite of the range of sampling depth from $1.5-7.0 \mathrm{~cm}$, the measurements of placental VTQ at the anterior and lateral uterus were similar (Fig. 3). The measurements of placental VTQ in this study $(0.983 \pm 0.260$ $\mathrm{m} / \mathrm{sec}$ ) were lower than those of the uterine endometrium $(2.05 \pm 0.77 \mathrm{~m} / \mathrm{sec})$ and uterine myometrium $(2.82 \pm 0.77 \mathrm{~m} / \mathrm{sec})$ [12], the parenchyma of the breast $(3.23 \pm 0.74 \mathrm{~m} / \mathrm{sec})$ [8], the child liver $(1.19 \pm 0.04 \mathrm{~m} / \mathrm{sec})[9]$, and the pancreas $(1.18 \pm 0.23 \mathrm{~m} /$ sec and $1.21 \pm 0.20 \mathrm{~m} / \mathrm{sec}$ ) [10]. These indicate that the placenta is a very soft organ. Our placental SWV findings are consistent with the results recently published by Ohmaru et al. [14]. The results of the present study suggest that during the gestation and the maturation of the placenta, the elasticity of the placenta undergoes little change, even though the echogenic characteristics, size, and

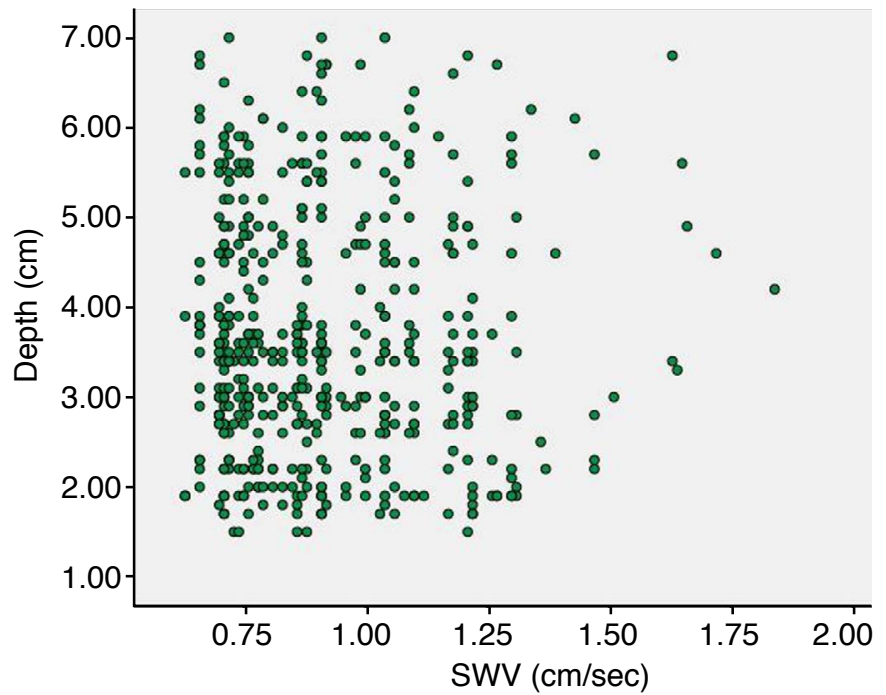

Fig. 3. Scatter plot of measurements of shear wave velocity (SWV) and depth of sample. The distribution of measurements of SWV is uneven at the same depth of sample.

volume of the placenta change over time [15-18]. Our study results showed that the elasticity of the normal placenta experiences little change in the second and third trimester; therefore, we infer that if placenta elasticity changes markedly during the pregnancy, there may be an abnormality in the placenta, the mother, or the fetus. We believe that the elasticity of the normal placenta may be useful as a reference parameter for the assessment of placental integrity in the future. Sugitani et al. [13] and Ohmaru et al. [14] reported that the value of VTQ was higher in placentas with fetal growth retardation, and its performance in placentas with other pathologies remains to be investigated. Research has identified methods for assessing the placenta, such as Grannum grades, and the VTQ technique may serve as a new addition to these methods [11,13-18].

In this study, we assumed that the ARFI technique was not harmful to the placenta and fetus based on the results of previous studies $[18,19]$. The study by Liu et al. [20], through a thermal safety assessment, indicates that thermal dose values, especially at the absorptive bone/soft tissue interface, could approach recommended dose thresholds if the cooling interval of multiple-frame ARFI elastography is too short. However, in clinical practice, ARFI elastography is of limited duration. In this study, the acting time of mechanical force ARFI for acquiring a time of VTQ by measurement of the SWV value $(\mathrm{m} / \mathrm{sec})$ is not more than 5 seconds. If seven valid measurements of SWV were performed in each placenta (as addressed in the methods of this article), ARFI may be triggered 21 to 30 times in total. The minimal interval between measurements was more than 2 seconds, the ARFI acted on seven discrete locations of the placenta, and the thermal dose values did not exceed 
recommended dose thresholds. Therefore, the VTQ technique is unlikely to do harm to the fetus. Further study and observation may be required in the future if people intend to use VTQ to evaluate the fetus.

Collectively, the results of this study show that the SWV of normal placenta tissue is $0.983 \pm 0.260 \mathrm{~m} / \mathrm{sec}$, it varies little between the second tand third trimesters, and the VTQ technique has potential for playing an additional role in the evaluation of the placenta.

ORCID: Size Wu: http://orcid.org/0000-0002-1086-764X; Ruixia Nan: http://orcid. org/0000-0002-9493-9638; Xiaojing Cui: http://orcid.org/0000-0003-0330-7772; Xian Liang: http://orcid.org/0000-0002-0175-3499

\section{Conflict of Interest}

No potential conflict of interest relevant to this article was reported.

\section{Acknowledgments}

This study was supported by the Scientific Research Foundation of the Hainan Provincial Health and Family Planning Commission (2014-51).

\section{References}

1. Guttmacher AE, Maddox YT, Spong CY. The Human Placenta Project: placental structure, development, and function in real time. Placenta 2014;35:303-304.

2. Chen $K H$, Chen LR, Lee YH. The role of preterm placental calcification in high-risk pregnancy as a predictor of poor uteroplacental blood flow and adverse pregnancy outcome. Ultrasound Med Biol 2012;38:1011-1018.

3. Chen KH, Chen LR, Lee YH. Exploring the relationship between preterm placental calcification and adverse maternal and fetal outcome. Ultrasound Obstet Gynecol 2011;37:328-334.

4. Nguyen D, Nguyen C, Yacobozzi M, Bsat F, Rakita D. Imaging of the placenta with pathologic correlation. Semin Ultrasound CT MR 2012;33:65-77.

5. Shiina T, Nightingale KR, Palmeri ML, Hall TJ, Bamber JC, Barr RG, et al. WFUMB guidelines and recommendations for clinical use of ultrasound elastography: Part 1: basic principles and terminology. Ultrasound Med Biol 2015;41:1126-1147.

6. Doherty JR, Trahey GE, Nightingale KR, Palmeri ML. Acoustic radiation force elasticity imaging in diagnostic ultrasound. IEEE Trans Ultrason Ferroelectr Freq Control 2013;60:685-701.

7. Lubinski MA, Emelianov SY, O'Donnell M. Speckle tracking methods for ultrasonic elasticity imaging using short-time correlation. IEEE Trans Ultrason Ferroelectr Freq Control 1999;46:82-96.
8. Golatta M, Schweitzer-Martin M, Harcos A, Schott S, Junkermann H, Rauch $G$, et al. Normal breast tissue stiffness measured by a new ultrasound technique: virtual touch tissue imaging quantification (VTIQ). Eur J Radiol 2013;82:e676-e679.

9. Fontanilla $T$, Canas T, Macia A, Alfageme M, Gutierrez Junquera C, Malalana A, et al. Normal values of liver shear wave velocity in healthy children assessed by acoustic radiation force impulse imaging using a convex probe and a linear probe. Ultrasound Med Biol 2014;40:470-477.

10. Xie J, Zou L, Yao M, Xu G, Zhao L, Xu H, et al. A preliminary investigation of normal pancreas and acute pancreatitis elasticity using virtual touch tissue quantification (VTQ) imaging. Med Sci Monit 2015;21:1693-1699.

11. Li WJ, Wei ZT, Yan RL, Zhang YL. Detection of placenta elasticity modulus by quantitative real-time shear wave imaging. Clin Exp Obstet Gynecol 2012;39:470-473.

12. Soliman AA, Wojcinski S, Degenhardt F. Ultrasonographic examination of the endometrium and myometrium using acoustic radiation force impulse (ARFI) imaging technology: an initial experience with a new method. Clin Hemorheol Microcirc 2015;59:235-243.

13. Sugitani M, Fujita Y, Yumoto Y, Fukushima K, Takeuchi T, Shimokawa $M$, et al. A new method for measurement of placental elasticity: acoustic radiation force impulse imaging. Placenta 2013;34:10091013.

14. Ohmaru T, Fujita Y, Sugitani M, Shimokawa M, Fukushima K, Kato K. Placental elasticity evaluation using virtual touch tissue quantification during pregnancy. Placenta 2015;36:915-920.

15. Sau A, Seed P, Langford $K$. Intraobserver and interobserver variation in the sonographic grading of placental maturity. Ultrasound Obstet Gynecol 2004;23:374-377.

16. Moran M, Ryan J, Higgins M, Brennan PC, McAuliffe FM. Poor agreement between operators on grading of the placenta. J Obstet Gynaecol 2011;31:24-28.

17. Chen CY, Su HW, Pai SH, Hsieh CW, Jong TL, Hsu CS, et al. Evaluation of placental maturity by the sonographic textures. Arch Gynecol Obstet 2011;284:13-18.

18. Yin TT, Loughna P, Ong SS, Padfield J, Mayhew TM. No correlation between ultrasound placental grading at 31-34 weeks of gestation and a surrogate estimate of organ function at term obtained by stereological analysis. Placenta 2009;30:726-730.

19. Palmeri ML, Frinkley KD, Nightingale KR. Experimental studies of the thermal effects associated with radiation force imaging of soft tissue. Ultrason Imaging 2004;26:100-114.

20. Liu Y, Herman BA, Soneson JE, Harris GR. Thermal safety simulations of transient temperature rise during acoustic radiation force-based ultrasound elastography. Ultrasound Med Biol 2014;40:1001-1014. 